\title{
Calcium pyrophosphate deposition and crowned dens syndrome
}

\begin{abstract}
C Alcium Pyrophosphate Deposition DisEAse (CPDD) was first described in the early 1960 s in patients with acute inflammatory arthritis that looked like gout. This was just after the development of the polarized light microscope and before the common use of morphologic or birefringent properties to identify crystal types. Astute clinician-scientists noticed that some of the synovial fluid crystals from patients with goutlike arthritis were resistant to uricase, and later discovered that these uricase-resistant crystals were composed of calcium and pyrophosphate.
\end{abstract}

\section{syndrome is an} underrecognized cause of acute neck pain and fever that is one of the more distinctive syndromes associated with CPDD

\section{See related article, page 204}

A description of a group of arthritis patients with radiographic chondrocalcinosis was also published around this time. Chondrocalcinosis appearing as finely stippled radiopaque deposits was shown to be a radiographic correlate of calcium pyrophosphate crystal deposition in tissues.

CPDD is still most commonly recognized as an acute inflammatory arthritis, formerly known as pseudogout. However, it has multiple clinical presentations, including a rheumatoid arthritis-like syndrome characterized by persistent inflammation of multiple large and small joints, as well as an osteoarthritis-like syndrome, with symptoms that are primarily degenerative but with a pattern of affected joints unusual for osteoarthritis. For example, shoulders, wrists, ankles, and metacarpophalangeal joints are commonly involved in CPDD, but this is relatively rare in osteoarthritis.

doi:10.3949/ccjm.88a.21008

\section{A NOT-SO-UNCOMMON FORM OF ARTHRITIS}

While the true incidence and prevalence of CPDD are unknown, it is not an uncommon form of arthritis. Estimates of prevalence suggest that 10 to 15 million Americans have some evidence of this disease. It is typically associated with advanced age and is rare in people under age 60. Familial forms are known to cause premature severe disease. Conditions that predispose to CPDD include hyperparathyroidism, hemochromatosis, hypomagnesemia, and hypophosphatasia.

Factors that govern crystal formation and control their ability to induce inflammation remain poorly characterized. Consequently, no specific therapies to prevent crystal formation or dissolve existing crystals have been designed to date. Moreover, CPDD lacks specific therapeutic recommendations, as there are virtually no randomized clinical trials of this disease. We treat the acute inflammatory syndromes in a manner similar to acute gout, and we struggle to find effective therapies for patients with the more chronic forms of this disease.

\section{LINK TO CROWNED DENS SYNDROME}

Crowned dens syndrome is an underrecognized cause of acute neck pain and fever that is one of the more distinctive clinical syndromes associated with CPDD.

In crowned dens syndrome, calcium pyrophosphate crystals deposit in the soft tissues around the dens. Affected patients are typically over the age of 65 and often have signifi- 
cant leukocytosis and dramatically elevated inflammatory markers. Delirium is also well described in these elderly patients.

Crowned dens syndrome is often misdiagnosed as meningitis or giant cell arteritis, and more rarely as polymyalgia rheumatica, spondylitis, or fever of unknown origin. The diagnosis is typically made by observing chondrocalcinosis surrounding the odontoid process on computed tomography of the cervical spine. Symptoms generally respond well to moderate or high doses of corticosteroids. Colchicine may be helpful, and cytokine-targeted anti-inflammatory drugs such as anakinra have also been used. ${ }^{1}$

\section{CHALLENGES TO DIAGNOSIS}

Crowned dens syndrome is considered rare, but little about its epidemiology is known, and it is likely that many cases are missed. Its diagnosis relies heavily on appropriate imaging. However, as in the case presented in this issue of the Journal by Mizumoto et al, ${ }^{2}$ a hasty diagnosis of crowned dens syndrome may result in missing a serious infection.

Conventional radiography and magnetic resonance imaging, often performed for musculoskeletal pain, will miss calcification around the dens. There are case reports of

\section{REFERENCES}

1. Aouba A, Deshayes S, Frenzel L, et al. Efficacy of anakinra for various types of crystal-induced arthritis in complex hospitalized patients: a case series and review of the literature. Mediators Inflamm 2015; 2015:792173. doi:10.1155/2015/792173

2. Mizumoto J. Crowned dens syndrome: caution about asymptomatic calcification. Cleve Clin J Med 2021; 88(4):204-205. doi:10.3949/ccjm.88a.20184

3. Heck A, Nolan N, Rojas-Moreno C. Crowned dens aspiration of local fluid collections that demonstrate calcium pyrophosphate crystals in crowned dens syndrome, ${ }^{3}$ but aspirations are rarely performed.

Conversely, the presence of calcific deposits in the periodontal ligament is not specific for the clinical symptoms of crowned dens syndrome and can occur in asymptomatic patients. This was illustrated nicely in a study ${ }^{4}$ of 513 patients undergoing computed tomography for acute trauma; the authors reported an overall $12.5 \%$ prevalence of atlantoaxial calcification, which increased to $34 \%$ in patients age 60 and older, and to $49 \%$ in those age 80 and older.

Improvements in imaging such as dual-energy computed tomography, which can chemically identify crystal deposits, will certainly aid in diagnosis. But until this type of test is widely available, increased clinical recognition of crowned dens syndrome as an important cause of acute neck pain with inflammatory signs and symptoms in older patients is essential.

\section{DISCLOSURES}

This work is supported by the Department of Veterans Affairs, Veterans Health Administration, Office of Research and Development (Merit Review Grant I01BX004454 (AKR). The views expressed in this article are those of the authors and do not necessarily reflect the position or policy of the Department of Veterans Affairs or the United States government.

syndrome: calcium pyrophosphate deposition disease masquerading as osteomyelitis. J Rheumatol 2018; 45(10):1422-1423. doi:10.3899/jrheum.170921

4. Chang EY, Lim WY, Wolfson T, et al. Frequency of atlantoaxial calcium pyrophosphate dihydrate deposition at CT. Radiology 2013; 269(2):519-524. doi:10.1148/radiol.13130125

Address: Ann K. Rosenthal, MD, Department of Medicine, Medical College of Wisconsin, 9200 W. Wisconsin Avenue, Milwaukee, WI 53226; arosenthal@mcw.edu 